\title{
Fetal brain MRI findings and neonatal outcome of common diagnosis at a tertiary care center
}

\author{
Monica S. Arroyo ${ }^{1,2} \cdot$ Robert J. Hopkin ${ }^{3,4} \cdot$ Usha D. Nagaraj ${ }^{4,5} \cdot$ Beth Kline-Fath $^{4,5} \cdot$ Charu Venkatesan $^{1,4}$
}

Received: 23 January 2019 / Revised: 10 April 2019 / Accepted: 10 May 2019 / Published online: 18 June 2019

(c) The Author(s), under exclusive licence to Springer Nature America, Inc. 2019

\begin{abstract}
:
Fetal Magnetic Resonance Imaging (MRI) is increasingly used in prenatal evaluations.

Objective: Identify common brain malformations on fetal MRI and evaluate perinatal course.

Methods: Fetal consultations from 10/2016 to 12/2017 reviewed.

Results: Hundred consultations were requested; 94 were completed. Findings included: posterior fossa malformations (19\%), agenesis/dysgenesis of corpus callosum (15\%), congenital aqueductal stenosis (CAS) (14\%), ventriculomegaly $(11 \%)$, isolated cortical malformations (8.5\%), and holoprosencephaly (6\%). Posterior fossa malformations were more likely to be associated with genetic conditions and cardiac malformations. Patients with CAS all required intensive care unit admission. Overall, few patients with congenital brain malformations required feeding or respiratory support at discharge. None had seizures as neonates except two with early epileptic encephalopathy syndromes.

Conclusions: Even though long term neurological prognosis is poor for many conditions including high lifetime risk of epilepsy, most are discharged with no feeding or respiratory support. Seizures are rarely seen in the neonatal period.
\end{abstract}

\section{Introduction}

Routine prenatal care includes surveillance for fetal anatomic malformations using ultrasound. Abnormalities detected via ultrasound often prompt a referral for a fetal magnetic resonance imaging (MRI) study, which can help clarify diagnosis [1-3]. Central nervous system malformations (CNS) account for a large proportion of consultations. In order to facilitate accurate prognostication, it is important to study both perinatal and long term outcomes of patients

Charu Venkatesan

charulata.venkatesan@cchmc.org

1 Division of Neurology, Cincinnati Children's Hospital, Cincinnati, OH, USA

2 Division of Pediatric Neurology, Joe DiMaggio Children's Hospital, Hollywood, CA, USA

3 Division of Human Genetics, Cincinnati Children's Hospital, Cincinnati, OH, USA

4 Department of Pediatrics, University of Cincinnati College of Medicine, Cincinnati, OH, USA

5 Division of Radiology and Medical Imaging, Cincinnati Children's Hospital, Cincinnati, OH, USA with prenatally diagnosed CNS malformations. Most studies to date have focused on long term outcome of patients with congenital brain malformations. The purpose of this study was to review fetal neurology consultations at a large tertiary medical center with a dedicated Fetal Care Center to evaluate the perinatal outcome and neonatal course of commonly encountered malformations. This information can guide family and providers in planning delivery and care in the immediate neonatal period.

\section{Methods}

We conducted a retrospective chart review of all fetal neurology consultations performed from 10/2016 to 12/ 2017 under Institutional Review Board approval (2018-1800). Nearly all fetal MRI scans were performed on 1.5 Tesla on site clinical magnets (GE Healthcare, Philips Healthcare) and were interpreted by one of 4 board certified pediatric radiologists with sub-specialty practice in fetal imaging. A routinely implemented fetal brain MRI protocol was implemented for which the majority of scan time was focused on acquiring T2-single shot fast spin echo images of the brain in three orthogonal planes, each repeated at least once to the radiologists' satisfaction. Maternal and 
neonatal charts were reviewed for clinical and demographic information.

\section{Results}

In the study time frame of 14 months, 100 neurology consults were requested. Six were cancelled (4 normal MRI; 1 fetal demise prior to meeting; 1 scheduling conflict); with 94 consults completed for review. The average maternal age was 27.7 years. The majority of the patients $(68 \%)$ reported no pregnancy complications. Substance use was noted in $16 \%$, hypertension in $12 \%$, and diabetes in $6 \%$. Amniocentesis was performed in $34 \%$ of patients. There were nine sets of twins in the cohort but only one set had CNS abnormalities in both fetuses.

Infants were classified according to primary malformation identified in fetal MRI. The most common findings were: posterior fossa malformations (19\%), agenesis/dysgenesis of corpus callosum (15\%), congenital aqueductal stenosis (14\%), ventriculomegaly without aqueductal stenosis $(11 \%)$, isolated cortical malformations (8.5\%), and holoprosencephaly $(6 \%)$ (Table 1$)$. When compared to postnatal findings, the primary fetal diagnostic classification remained unchanged in $98 \%$ of cases. In one case, diagnosis changed from meningocele prenatally (34 4/7 weeks) to encephalocele postnatally. One patient was diagnosed with severe ventriculomegaly suggestive of aqueductal stenosis at $213 / 7$ weeks gestation. However, postnatally, images showed mild ventriculomegaly with narrowing of the ventral cerebral aqueduct and hypogenesis of the corpus callosum. Minor changes that did not alter the fetal finding and counseling were noted on post-natal imaging; these included evolution of intracranial injury and detection of additional intracranial abnormalities such as cortical malformations (most commonly, heterotopias).

\section{Posterior fossa malformations}

A total of 18 patients had posterior fossa malformations (Table 1). One pregnancy with diagnoses of Joubert syndrome, Dandy-Walker malformation and polycystic kidneys was terminated, resulting in 17 live births. The more commonly seen diagnoses included Dandy-Walker Malformation $(n=4)$ (Fig. 1a), vermian hypoplasia $(n=3)$ and Chiari III malformation $(n=2)$; other diagnoses included rhombencephalosynapsis with mild ventriculomegaly, suboccipital meningocele, mega cisterna magna, pontocerebellar hypoplasia, Chiari II malformation, Blake pouch remnant, and Joubert syndrome (Table 1). Seven patients had post-natal genetic testing (four had microarray, two had chromosome analysis and one had Joubert panel analysis). This confirmed a diagnosis of Turner's syndrome in a
Table 1 Summary of common brain malformations on fetal MRI

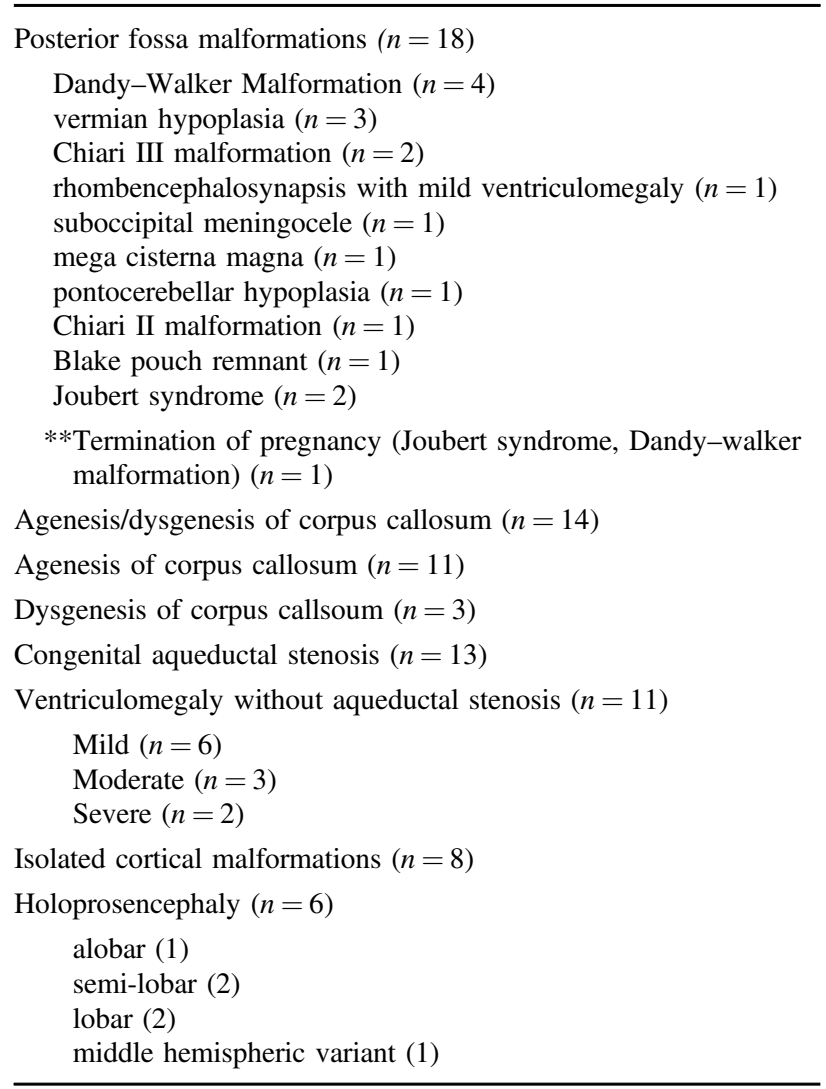

patient with vermian hypoplasia and Down's syndrome in a patient with Dandy-Walker continuum.

Two families opted for palliative care and both babies died a few hours after birth: one fetus was diagnosed with pontocerebellar hypoplasia and another one was diagnosed with Dandy-Walker spectrum and Pierre Robin sequence. Of the remaining 15 infants, $67 \%$ were born at term. Only three patients required intubation in the delivery room, with two patients having genetically confirmed CHARGE syndrome (coloboma, heart defects, choanal atresia, mental retardation, genital hypoplasia, and ear abnormalities) and a third patient with Chiari III malformation and micrognathia. Thirteen out of 15 patients were admitted to the ICU and the average ICU stay was 26 days. Four patients had cardiac anomalies including coarctation of the aorta, CHARGE syndrome and a mitral valve cleft. Both patients diagnosed with CHARGE syndrome died in the ICU after four days of life.

In terms of respiratory support, one of the patients with Chiari III malformation required tracheostomy tube placement. One patient with Joubert syndrome required oxygen at discharge for apnea. Longer term feeding assistance was required in three patients: one born prematurely with Dandy-Walker malformation and coarctation of the aorta 


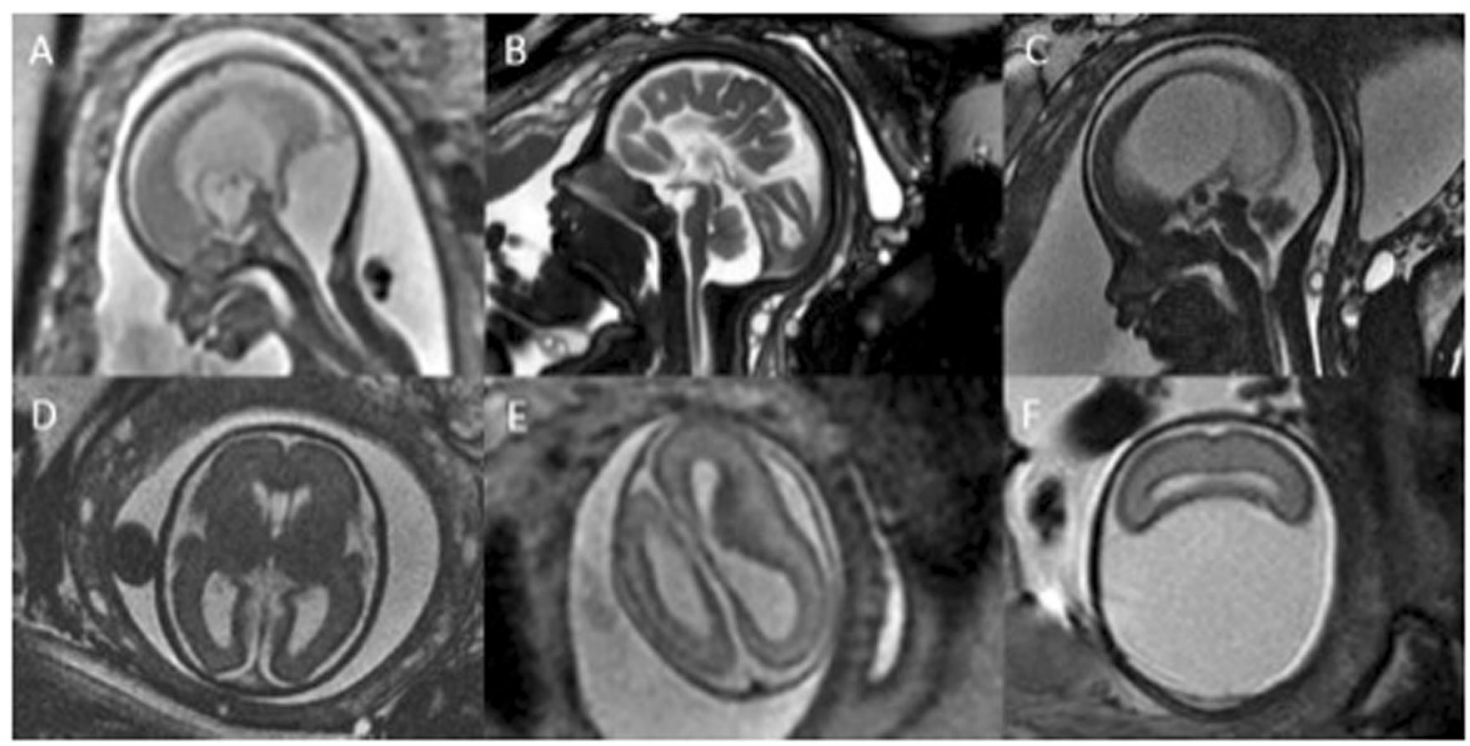

Fig. 1 a Sagittal T2-SSFSE image from fetal MRI of a 21 week 4 days gestational age fetus with posterior fossa findings consistent with Dandy-Walker malformation with associated obstructive hydrocephalus. b Sagittal FIESTA/bFFE image from fetal MRI of a 34 weeks gestational age fetus with complete Agenesis of the Corpus Callosum. c Sagittal FIESTA/bFFE image from fetal MRI of a 26 weeks gestational age fetus with obstructive hydrocephalus secondary to Aqueductal Stenosis. d Axial FIESTA/bFFE image from fetal MRI of a 27 week 3 days gestational age fetus with mild Ventriculomegaly of the lateral ventricles, without other structural brain abnormalities identified. e Axial T2-SSFSE image from fetal MRI of a 20 weeks gestational age fetus with left Hemimegalencephaly. f Axial T2-SSFSE image from a fetal MRI of a 25 weeks 1 day gestational age fetus with alobar Holoprosencephaly with an associated dorsal cyst and both patients with Chiari III malformation. The rest were discharged on full oral feeds and have not yet required any additional feeding assistance, with ages ranging $1-13$ months.

\section{Congenital aqueductal stenosis}

A total of 13 fetuses were diagnosed with congenital aqueductal stenosis (Fig. 1c) (Table 1). Two also had rhombencephalosynapsis. Seven mothers underwent amniocentesis and three had sampling of free fetal DNA with one diagnosis of Trisomy 21; this fetus had in utero demise at 23 weeks. Two pregnancies were terminated and one baby was lost to follow up. Neonatal course was available for nine babies for review.

All nine were born after 37 weeks and none required intubation in the delivery room. All required ICU stay with an average duration of 30 days. Seven patients required placement of intracranial shunt in the ICU. One patient showed resolution of ventriculomegaly on postnatal MRI. An additional patient required a shunt at 3 months of age. No patients had seizures in the neonatal period. Two of the five males had L1CAM mutation detected post-natally and both of these patients had adducted thumbs seen on fetal MRI. No additional genetic syndromes were identified. Three patients were discharged with NG feeding and eventually only one of them required G-tube. This patient passed away at 4 months for unknown reasons. Only one baby required oxygen nasal cannula support at discharge for obstructive sleep apnea.

\section{Ventriculomegaly without aqueductal stenosis}

A total of 11 fetuses had isolated ventriculomegaly, which was classified as mild in 6/11 (10-12 mm; Fig. 1d), moderate in 3/11 (>12-15 mm; two were twins) and severe in $2 /$ 11 (>15 mm) (Table 1). There were no pregnancy terminations. Postnatal information was missing on one baby; $50 \%$ of infants were born via cesarean section. No patients required intubation at delivery. Only three patients required ICU stay: one for complex cardiac malformation and two for respiratory causes (one for 2 days and one for 15 days). The patient with the cardiac malformation passed away in the ICU. None of the surviving patients in the cohort required respiratory or feeding support at discharge.

\section{Agenesis of the corpus callosum spectrum}

There were 14 fetal consults for agenesis or dysgenesis of the corpus callosum (Fig. 1b) (Table 1). Eleven out of fourteen patients had complete agenesis of the corpus callosum (AgCC) while three had dysgenesis of the CC. Of the 11 patients with $\mathrm{AgCC}$, five had isolated $\mathrm{AgCC}$ while the 
remainder had other accompanying intracranial malformations. Among the three patients with callosal dysgenesis, one was later diagnosed with Aicardi syndrome clinically. Another patient with callosal dysgenesis had a large deletion of chromosome 18 discovered on prenatal amniocentesis with kidney and cardiac involvement; this baby passed away at 4 months of age. There were no pregnancy terminations. Detailed postnatal course was available for 11 patients. Seven babies were admitted to the ICU and five of these admissions lasted less than 5 days. Two babies were admitted for $\sim 3$ months; one had the large deletion of chromosome 18 and the other patient had accompanying left frontal lobe polymicrogyria. This latter infant required prolonged hospitalization for feeding support; he was able to feed orally within the first year of life and Gtube was removed. None of the surviving patients required respiratory support at discharge. No patient has required treatment for endocrine deficiency (infants between 10 days and 15 months).

\section{Holoprosencephaly}

Six patients were diagnosed with holoprosencephaly (HPE) on fetal imaging (Table 1). One had alobar (Fig. 1f), two had semi-lobar, two had lobar and 1 had middle hemispheric variant. All underwent some type of prenatal genetic testing; two had free fetal DNA analysis (normal) and four had amniocentesis (three had normal FISH and microarray; one was noted to have a deletion of a portion of chromosome 13). Postnatal testing confirmed a deletion in 13q32.2$\mathrm{q} 34$, which includes the ZIC2 gene. This gene abnormality is associated with microcephaly and complex brain malformations, including middle interhemispheric fusion and posterior encephalocele.

The patient with alobar HPE was discharged home with NG feeding after a 13 day stay and was transitioned to full oral feeding at home. She is 7 months of age presently and has not required placement of intraventricular shunt or respiratory support. Endocrine evaluation has been unremarkable thus far. One patient with semi-lobar HPE had significant cardiac anomalies and died in the cardiac ICU at 5 days of life after family elected palliative/comfort care. The other patient with semi-lobar holoprosencephaly had multiple facial anomalies including bilateral cleft lip and palate; this patient had placement of G-tube and tracheostomy tube during a NICU stay of 30 days. One patient with lobar HPE was found to have Cornelia de Lange syndrome and pregnancy was terminated. Post-natal information was missing for the second patient with lobar holoprosencephaly. Lastly, the patient with the middle hemispheric variant form of holoprosencephaly and large deletion in chromosome 13q had ventriculoperitoneal shunt placement at about 5 weeks of age following discharge from the NICU and he has not required external feeding or respiratory assistance.

\section{Seizures in the neonatal period}

Patients with the most commonly seen diagnoses (posterior fossa malformations, venticulomegaly with or without aqueductal stenosis, agenesis of the corpus callosum) did not have clinical seizures in the neonatal period. Decision to perform EEG was based on clinical indication by treating physician. EEG studies were available for 13 patients. In our study, only two patients had seizures in the neonatal period. The first was a patient with microcephaly and simplified gyral pattern who was found to harbor a FOXG1 mutation post-natally; he developed seizures within the first week of life while admitted to the NICU for respiratory issues due to choanal atresia. The second patient had hemimegalencephaly (Fig. 1e) in the setting of an overgrowth syndrome suspected to be a PIK3CA-related overgrowth spectrum disorder; she had intractable seizures in the NICU.

\section{Discussion}

MRI is increasingly used in the evaluation of CNS anomalies in the fetus. Sub-specialists including neonatologists, obstetricians, neurologists and geneticists are asked to provide short and long term prognostic information to families. A large study comparing 242 fetal ultrasound and fetal MRI studies showed that MR imaging changed diagnosis in about $32 \%$ of patients [4]. Large multicenter trials have shown that fetal MRI can have a diagnostic accuracy of $93 \%$ while ultrasound was found to have an accuracy of 68\%. [5] Additionally, it has been shown that women who undergo fetal MRI found the study to be valuable with $95 \%$ reporting that they would undergo fetal MRI if a future pregnancy was noted to have a central nervous system abnormality [5].

There are currently limited data on the immediate perinatal and neonatal outcomes of these patients, which makes counseling inherently challenging. In order to optimize care and provide a more accurate clinical picture to families, we wanted to explore the immediate neonatal outcome of babies with prenatally diagnosed brain anomalies. This information can assist providers in delivery planning (e.g. need for transfer to higher level of ICU care after birth) and families' expectations after birth.

In our retrospective study of fetal consultations, diagnosis was accurate and remained unchanged post-natally in $98 \%$ of patients. As expected, post- natal MRI identified additional foci of cortical malformations or progression of vascular injury, but these findings did not change the clinical implications that had already been discussed during fetal counseling. There were 2 cases in which the diagnosis significantly changed between the fetal MRI and the post- 
natal MRI. One fetus was diagnosed with severe ventriculomegaly at $213 / 7$ weeks gestation and post-natal imaging showed only mild ventriculomegaly and a narrowed ventral aspect of the cerebral aqueduct. It is possible that the degree of narrowing changed in a favorable manner in subsequent weeks of gestation to allow for adequate CSF flow; thus post-natal imaging reflected this positive structural change. Another fetus was diagnosed with meningocele pre-natally that was post-natally found to be an encephalocele. It is possible that the brain herniated further into the cavity through pregnancy. There are limitations to prenatal imaging and the possible presence of brain tissue within the cyst should always be discussed in fetal counseling.

Based on our findings, we recommend postnatal MRI in the neonatal period when potential neurosurgical intervention is anticipated (e.g congenital aqueductal stenosis, Dandy-Walker malformation, holoprosencephaly, moderate or severe ventriculomegaly). Patients with mild ventriculomegaly may be followed by head ultrasound initially. MRI can be performed more urgently if clinical concerns about unexpected head growth occur. In patients with identified or suspected cortical malformations, imaging can be deferred until they are over 15 months of age when myelination is more optimal to allow for detailed imaging unless unexpected clinical concerns arise. A similar approach can also be used for patients with callosal agenesis/dysgenesis without intracranial cysts.

Overall, seizures occurred rarely in the neonatal period. In fact, in our study cohort, only two patients had seizures in the neonatal period. The first patient had microcephaly and cortical malformation due to FOXG1 mutation. The second patient had hemimegalencephaly in the setting of an overgrowth syndrome. Both conditions are associated with an epileptic encephalopathy phenotype and carry a high risk of neonatal seizures. Thus, while there is a very high lifetime risk of epilepsy in patients with intracranial malformations, clinical seizures were not typically noted in the neonatal period. However, the retrospective nature of our study limits our ability to comment on potential subclinical seizures and interictal EEG abnormalities in patients in the neonatal period.

Malformations affecting the posterior fossa were the most common type seen in our cohort of fetal consultations. A limitation of our study was that it only covered consultations performed by Pediatric Neurologists. At our institution, Pediatric Neurologists are not involved in prenatal consultations of spinal cord pathology. As such, the focus of this study is on brain malformations. Compared to other CNS malformations, posterior fossa malformations were more likely to be associated with genetic conditions including CHARGE, Down's and Turner's syndromes and these patients had associated cardiac malformations. The association between cardiac malformations and these genetic syndromes has been well described [6]. A published cohort of 24 patients with Dandy-Walker malformation found cardiac malformations in $44 \%$ of patients [7]. Our study suggests that given the high rate of systemic complications accompanying posterior fossa malformations, these babies should be delivered in hospitals with NICU capability to provide supportive care and engage in additional investigations and subspecialists. Interestingly, the vast majority of surviving patients did not require respiratory or feeding support at discharge.

Congenital aqueductal stenosis was diagnosed in $15 \%$ of patients. Despite significant ventriculomegaly noted on fetal MRI in patients with congenital aqueductal stenosis, all of the patients were born at term gestation and none required intubation in the delivery room. However, majority had shunts placed in the neonatal period. Therefore, it is important for these babies to be delivered at a site where neurosurgical consultation services are readily available. Despite the striking imaging findings that can alarm families, only one infant required oxygen via nasal canula at discharge for obstructive sleep apnea and one infant required longer term feeding assistance via G-tube.

There were 6 patients with a diagnosis of holoprosencephaly. Classification of HPE is based on the degree of separation of the cerebral hemispheres; these include alobar, semi-lobar, lobar and middle interhemispheric variant, [8] outcome is worst for alobar HPE and carries a high mortality rate [8, 9]. HPE is also associated with chromosomal anomalies and systemic complications [8, 10, 11]. In our study, neonatal outcome was determined by accompanying malformations. Our experience suggests that during fetal counseling, it is important to emphasize that even patients with alobar holoproscencephaly can survive the neonatal period with minimal support. Given the very poor long term prognosis, especially with alobar holoprosencephaly, families may be inappropriately counseled that the infant will certainly die soon after birth. This leaves families inadequately prepared for future decision-making when the baby not only survives, but requires minimal external ventilator and feeding support at birth.

Agenesis of the corpus callosum was seen in isolation and as part of other CNS malformations. Patients did well overall. This aligns with long term data which show patients with isolated agenesis of the corpus callosum typically have minor deficits including language delays, learning disabilities and fine motor difficulties [12-14]. In our study we found that only patients with additional malformations beside $\mathrm{AgCc}$ needed to have access to ICU level care after delivery. One of the female patients with AgCC also had inter hemispheric cysts and developed infantile spasms at 2 months of age. Postnatal ophthalmological evaluation confirmed the presence of bilateral chorioretinal lacunae. 
She was diagnosed with Aicardi syndrome, suggesting that females with $\mathrm{AgCC}$ and intracranial cysts detected prenatally should receive appropriate counseling regarding this diagnosis.

Fetal MRI is being used with increasing frequency to assist with prognostication of neurological conditions and to guide families and providers in panning of immediate postnatal care. Our study finds that invasive respiratory support is not needed in the immediate neonatal period with most brain malformations, even in conditions that are typically associated with very poor long term outcome. Given that the primary fetal diagnostic classification remained unchanged in $98 \%$ of cases, immediate post-natal imaging should be reserved for infants requiring surgical intervention; otherwise, timing and need for post-natal imaging should be determined by the nature of the brain malformation. Clinical seizures were rarely noted in the immediate neonatal period; however, future studies will need to critically assess the benefits of surveillance EEG monitoring in neonates with brain malformations.

\section{Compliance with ethical standards}

Conflict of interest The authors declare that they have no conflict of interest.

Publisher's note: Springer Nature remains neutral with regard to jurisdictional claims in published maps and institutional affiliations.

\section{References}

1. Verburg B, Fink AM, Reidy K, Palma-Dias R. The contribution of MRI after fetal anomalies have been diagnosed by ultrasound: correlation with postnatal outcomes. Fetal Diagn Ther. 2015;38:186-94.

2. Rossi AC, Prefumo F. Additional value of fetal magnetic resonance imaging in the prenatal diagnosis of central nervous system anomalies: a systematic review of the literature. Ultrasound Obstet Gynecol. 2014;44:388-93.

3. Kett JC, Woodrum DE, Diekema DS. A survey of fetal care centers in the United States. J Neonatal Perinat Med. 2014;7:131-5.

4. Levine D, Barnes PD, Robertson RR, Wong G, Mehta TS. Fast MR imaging of fetal central nervous system abnormalities. Radiology . 2003;229:51-61.

5. Griffiths PD, Bradburn M, Campbell MJ, Cooper CL, Graham R. MERIDIAN collaborative group et al. Use of MRI in the diagnosis of fetal brain abnormalities in utero (MERIDIAN): a multicentre, prospective cohort study. Lancet . 2017;389:538-46.

6. Oliveira PHA, Souza BS, Pacheco EN, Menegazzo MS, Corrêa IS, Zen PRG, et al. Genetic syndromes associated with congenital cardiac defects and ophthalmologic changes-systematization for diagnosis in the clinical practice. Arq Bras Cardiol. 2018;110:84-90.

7. Sasaki-Adams D, Elbabaa SK, Jewells V, Carter L, Campbell JW, Ritter AM. The Dandy-Walker variant: a case series of 24 pediatric patients and evaluation of associated anomalies, incidence of hydrocephalus, and developmental outcomes. J Neurosurg Pediatr. 2008;2:194-9.

8. Kauvar EF, Muenke M. Holoprosencephaly: recommendations for diagnosis and management. Curr Opin Pediatr Dec. 2010;22:687-95.

9. Kaliaperumal C, Ndoro S, Mandiwanza T, Reidy F, McAuliffe F, Caird J, et al. Holoprosencephaly: antenatal and postnatal diagnosis and outcome. Childs Nerv Syst. 2016;32:801-9.

10. Hu T, Kruszka P, Martinez AF, Ming JE, Shabason EK, Raam MS, et al. Cytogenetics and holoprosencephaly: a chromosomal microarray study of 222 individuals with holoprosencephaly. Am J Med Genet C Semin Med Genet. 2018;178:175-86.

11. Kruszka P, Muenke M. Syndromes associated with holoprosencephaly. Am J Med Genet C Semin Med Genet. 2018;178:229-37.

12. D'Antonio F, Pagani G, Familiari A, Khalil A, Sagies TL, Malinger G, et al. Outcomes associated with isolated agenesis of the corpus callosum: a meta-analysis. Pediatrics. 2016;138: e20160445.

13. Siffredi V, Anderson V, Leventer RJ, Spencer-Smith MM. Neuropsychological profile of agenesis of the corpus callosum: a systematic review. Dev Neuropsychol. 2013;38:36-57.

14. Siffredi V, Anderson V, Mcllroy A, Wood AG, Leventer RJ, Spencer-Smith MM. A Neuropsychological profile for agenesis of the corpus callosum? Cognitive, academic, executive, social, and behavioral functioning in school-age children. J Int Neuropsychol Soc. 2018;24:445-55. 